\title{
Soft leptogenesis in the NMSSM with a singlet right-handed neutrino superfield
}

\author{
Waleed Abdallah, ${ }^{a, b}$ Abhass Kumar ${ }^{c}$ and Abhijit Kumar Saha ${ }^{c}$ \\ ${ }^{a}$ Harish-Chandra Research Institute, \\ Chhatnag Road, Jhunsi, Allahabad 211 019, India \\ ${ }^{b}$ Department of Mathematics, Faculty of Science, Cairo University, \\ Giza 12613, Egypt \\ ${ }^{c}$ Theoretical Physics Division, Physical Research Laboratory, \\ Navrangpura, Ahmedabad 380 009, India \\ E-mail: waleedabdallah@hri.res.in, abhass@prl.res.in, aks@prl.res.in
}

ABSTRACT: In this work, we explore soft leptogenesis in the NMSSM framework extended by a right-handed neutrino superfield. We calculate the CP asymmetry, $\varepsilon$, and find it to be non-zero at tree-level without using thermal effects for the final state particles. This is in contrast to soft leptogenesis in the MSSM extended by a right-handed neutrino superfield where thermal effects are essential. The difference arises due to the presence of a 3-body decay of the sneutrino in the NMSSM that violates lepton number at tree-level. Apart from this, we also find that $\varepsilon \neq 0$ if the additional singlet scalar has a complex vacuum expectation value while all the other NMSSM parameters including the soft SUSY breaking ones relevant for CP asymmetry remain real. We estimate the order of magnitudes of these parameters to produce sufficient baryon asymmetry of the Universe.

KEYwords: Cosmology of Theories beyond the SM, CP violation, Supersymmetric Standard Model, Neutrino Physics

ARXIV EPRINT: 1911.03363 


\section{Contents}

1 Introduction 1

2 Model 3

2.1 Terms relevant for soft leptogenesis 3

3 CP asymmetry 4

$3.1 \tilde{N}$ decays 5

$3.2 \sigma$ decays 9

4 General constraints $\quad 10$

4.1 A simpler form for $\varepsilon \quad 11$

5 Results and discussions $\quad 11$

5.1 Case 1: $\delta$ is near zero 13

5.2 Case $2: \delta=\pi \quad 14$

6 Conclusion 14

\section{Introduction}

It is well known that the observable Universe has an asymmetry between baryons and antibaryons [1], often called the problem of baryogenesis. Over the years, many mechanisms have been proposed to create this baryon asymmetry. More recently, leptogenesis $[2,3]$ has become a highly favoured model for baryogenesis, specially because this mechanism is naturally linked to neutrino masses. Adding right-handed $(\mathrm{RH})$ singlet heavy neutrinos to the standard model (SM) generates neutrino masses by the seesaw mechanism [4-9]. These RH neutrinos can also decay to produce a scalar and SM leptons and if the decay violates $\mathrm{CP}$ for example due to interference between tree-level and loop-level decays owing to complex couplings, a lepton asymmetry is generated. Since, in the SM, the $B-L$ symmetry is exact while the $B+L$ symmetry is broken by the electroweak (EW) sphaleron processes [10], these sphaleron processes can convert the generated lepton asymmetry to baryon asymmetry.

Soft leptogenesis [11-13] pertains to generating lepton asymmetry at the tree-level itself due to mixing between the particle and anti-particle states of the RH singlet sneutrino, $\tilde{N}$, because of the presence of soft SUSY breaking terms. ${ }^{1}$ In the most minimal soft leptogenesis setup using minimal supersymmetric standard model (MSSM) $[25,26]$ extended by one RH neutrino superfield, $\hat{N}$, a $\mathrm{CP}$ asymmetry in the $\mathrm{RH}$ sneutrino sector is created only when

\footnotetext{
${ }^{1}$ Soft leptogenesis in different types of SUSY framework can be found in [14-24].
} 
thermal masses for the final products are considered. The asymmetry is present because the thermal phase space factors are different for bosons and fermions. Another work featuring soft leptogenesis looks at $\mathrm{CP}$ violation not just due to mixing between particle and antiparticle initial states but in decays and in the interference of mixing and decay [27]. In ref. [28], it is shown that considering most generic soft trilinear couplings and one loop self energy contributions for sneutrino decay it is possible to generate CP violation even without finite temperature effects within the same setup.

However, the MSSM suffers from the so-called $\mu$-problem [29] — there is no explanation to why the SUSY scale preserving $\mu$-term (a direct SUSY mass term for the Higgs fields) should be of the same order as the soft SUSY breaking terms. The most straightforward solution to the $\mu$-problem comes by promoting the $\mu$-parameter into a field whose vacuum expectation value (vev) is determined, like the other scalar field vevs, from the minimization of the scalar potential along the new field direction [30]. Naturally, it is expected to fall in the range of the other vevs, i.e., of order $\mathcal{O}\left(M_{\mathrm{SUSY}}\right)$. The next-to-minimal supersymmetric standard model (NMSSM) (for review see $[30,31]$ ) is the most simple and elegant model to solve this problem, where a singlet superfield $\hat{S}$ is introduced to the MSSM superfields which gets non-zero vev. The NMSSM can be extended by a set of RH neutrino superfields to generate masses for the SM light neutrinos by the type-I seesaw (see [32-34] for the MSSM extended by RH neutrino superfield). This has been explored earlier in ref. [35]. This extension also keeps the R-parity conserved if the sneutrinos do not get vevs [35].

In this work, by using the NMSSM extended by the RH neutrino superfield, we present a soft leptogenesis scenario that creates a lepton asymmetry at the tree-level decay of the $\mathrm{RH}$ sneutrino without using thermal mass factors. The $\mathrm{CP}$ violation is achieved by the mixing between the particle and anti-particle states. This is due to the presence of the soft terms and the trilinear coupling between the additional singlet superfield which takes a vev and the RH neutrino superfield. A similar non-SUSY setup with such a trilinear term can be found in [36]. We also show that it is possible to obtain non-zero CP asymmetry even when all the soft parameters are real. Since the soft terms are responsible for creating the CP asymmetry instead of needing flavour effects as in usual leptogenesis, using only one generation of the RH neutrino superfield is enough. Even so, the setup can be easily extended to get the experimentally observed SM neutrino mass hierarchies and their mixing angles pattern [37, 38].

The paper is organised in the following manner. We setup the model and segregate the parts required for soft leptogenesis in the next section (section 2). In the one following that, i.e., section 3, we calculate the CP asymmetry produced by decays of the various particle present in the model that contribute to non-zero CP asymmetry parameter $\varepsilon$ at the treelevel. We talk about the decays of $\tilde{N}$ as well as the scalar $S$ in the model. In section 4 , we discuss the most crucial and important constraints and give a simple expression for $\varepsilon$. In section 5, we give and discuss the results of our calculation. We find that for successfully generating the observed baryon asymmetry of the Universe, we need $\varepsilon \approx \mathcal{O}\left(10^{-6}\right)$. We also discuss what this could mean for various parameters of the model including the soft ones. We finally conclude in section 6 . 


\section{Model}

In the NMSSM, an extra singlet superfield $\hat{S}$ is added to the MSSM Higgs sector [30]. Assuming explicitly $\mathbb{Z}_{3}$ symmetry, the superpotential for the NMSSM with a singlet RH neutrino superfield $\hat{N}$ in terms of the new singlet superfield $\hat{S}$ and the MSSM doublet superfields $\hat{H}_{u}$ and $\hat{H}_{d}$ will be as follows [30]:

$$
W=Y_{E}^{i j} \hat{H}_{d} \hat{L}_{i} \hat{E}_{j}+Y_{D}^{i j} \hat{H}_{d} \hat{Q}_{i} \hat{D}_{j}+Y_{U}^{i j} \hat{H}_{u} \hat{Q}_{i} \hat{U}_{j}+\lambda \hat{S} \hat{H}_{u} \hat{H}_{d}+\frac{\kappa}{3} \hat{S}^{3}+Y_{N}^{i} \hat{N} \hat{H}_{u} \hat{L}_{i}+\lambda_{N} \hat{S} \hat{N} \hat{N}
$$

where $\hat{L}_{i}$ and $\hat{Q}_{i}$ are the $\mathrm{SU}(2)$ doublet superfields of leptons and quarks; $\hat{E}_{i}$ and $\hat{D}_{i}$ $\left(\hat{U}_{i}\right)$ denote singlet down (up)-type quark superfields, respectively, and $Y$ 's, $\lambda$ 's and $\kappa$ are dimensionless couplings with generation indices $(i, j=1,2,3)$. After the singlet $S$ obtains a vacuum expectation value (vev) $\langle S\rangle$, an effective $\mu$-term is generated: $\mu_{\text {eff }}=\lambda\langle S\rangle$, which solves the so-called $\mu$-problem [29]. The soft SUSY-breaking Lagrangian is given by

$$
\begin{aligned}
-\mathcal{L}_{\text {soft }}= & -\left.\mathcal{L}_{\text {soft }}^{\mathrm{MSSM}}\right|_{B \mu=0}+\left(A_{\lambda_{H}} \lambda S H_{u} H_{d}+A_{\kappa} \frac{\kappa}{3} S^{3}+A_{N}^{i} Y_{N}^{i} \tilde{N} H_{u} \tilde{L}_{i}+A_{\lambda} \lambda_{N} S \tilde{N} \tilde{N}+\text { h.c. }\right) \\
& +m_{S}^{2}|S|^{2}+M^{2}|\tilde{N}|^{2}
\end{aligned}
$$

where $\tilde{L}_{i}$ and $\tilde{N}$ are the scalar components of $\hat{L}_{i}$ and $\hat{N}$ superfields, respectively. CP is spontaneously violated when the scalars $H_{u}, H_{d}, S$ attain vevs with relative physical phases. The vev of the singlet $S$ is complex:

$$
\langle S\rangle=v_{S} e^{i \delta}
$$

Since leptogenesis occurs above the electroweak (EW) phase transition, we do not give vevs to the two Higgs doublets. In this case, spontaneous CP violation can occur only when $\sin \delta \neq 0$.

\subsection{Terms relevant for soft leptogenesis}

The terms from the superpotential required for leptogenesis via sneutrino decay are:

$$
W \supset Y_{N} \hat{L} \hat{H} \hat{N}+\lambda_{N} \hat{S} \hat{N} \hat{N}+\frac{\kappa}{3} \hat{S}^{3} .
$$

Here we consider $\lambda_{N}, \kappa$ to be all real and positive. We also remove the $i, j$ indices from the leptons and the $u$ index from the Higgs superfield for brevity. The scalar potential is obtained using:

$$
V_{S}=\left|\frac{\partial W}{\partial S}\right|^{2}+\left|\frac{\partial W}{\partial N}\right|^{2}+\left|\frac{\partial W}{\partial L}\right|^{2}
$$

with

$$
\begin{aligned}
& \left|\frac{\partial W}{\partial S}\right|^{2}=\lambda_{N}^{2}|\tilde{N}|^{4}+\kappa^{2}|S|^{4}+\lambda_{N} \kappa S^{* 2} \tilde{N} \tilde{N}+\lambda_{N} \kappa \tilde{N}^{*} \tilde{N}^{*} S^{2} \\
& \left|\frac{\partial W}{\partial N}\right|^{2}=\left|Y_{N}\right|^{2}|\tilde{L}|^{2}|H|^{2}+4 \lambda_{N}^{2}|\tilde{N}|^{2}|S|^{2}+2 \lambda_{N} Y_{N}^{*} \tilde{N} S \tilde{L}^{*} H^{*}+2 \lambda_{N} Y_{N} \tilde{N}^{*} S^{*} H \tilde{L} \\
& \left|\frac{\partial W}{\partial L}\right|^{2}=\left|Y_{N}\right|^{2}|H|^{2}|\tilde{N}|^{2}
\end{aligned}
$$


The fermionic part of the Lagrangian is given by:

$$
\mathcal{L}_{f}=Y_{N} L \tilde{H} \tilde{N}+\lambda_{N} S N N+Y_{N} L H N .
$$

The soft SUSY-breaking Lagrangian terms that play a role in leptogenesis are:

$$
-\mathcal{L}_{\text {soft }} \supset\left(A_{\kappa} \frac{\kappa}{3} S^{3}+A_{N} Y_{N} \tilde{L} H \tilde{N}+A_{\lambda} \lambda_{N} S \tilde{N} \tilde{N}+\text { h.c. }\right)+m_{S}^{2}|S|^{2}+M^{2}|\tilde{N}|^{2} .
$$

The superpotential and the soft breaking terms combine to give the following interactions for $\tilde{N}$ and $\sigma$ which could in principle contribute to soft leptogenesis due to mixing between the particle and anti-particle states through the soft terms:

$$
\begin{aligned}
\mathcal{L}_{\text {int }}= & \tilde{N}\left(Y_{N} \tilde{H} L+2 \lambda_{N} Y_{N}^{*} v_{S} e^{i \delta} H^{*} \tilde{L}^{*}+2 \lambda_{N} Y_{N}^{*} \sigma H^{*} \tilde{L}^{*}+A_{N} Y_{N} H \tilde{L}\right) \\
& +\sigma\left(\lambda_{N} N N+A_{\lambda} \lambda_{N} \tilde{N} \tilde{N}\right)+h . c .
\end{aligned}
$$

where $\sigma=S-\langle S\rangle$.

\section{CP asymmetry}

Because of the soft terms as well as the vev of $S$, there is a mixing between particle and anti-particle states of the sneutrino and the singlet scalar $\sigma$ which is the dynamic part of $S$. The squared mass matrices for the two of them are given by:

$$
\begin{aligned}
\mathcal{M}_{\tilde{N}}^{2} & =\left[\begin{array}{cc}
M_{1}^{2} & \lambda_{N} \kappa v_{S}^{2} e^{2 i \delta}+A_{\lambda} \lambda_{N} v_{S} e^{-i \delta} \\
\lambda_{N} \kappa v_{S}^{2} e^{-2 i \delta}+A_{\lambda} \lambda_{N} v_{S} e^{i \delta} & M_{1}^{2}
\end{array}\right], \\
\mathcal{M}_{\sigma}^{2} & =\left[\begin{array}{cc}
m_{\sigma}^{2} & 2 \kappa^{2} v_{S}^{2} e^{2 i \delta}+2 A_{\kappa}^{*} \kappa v_{S} e^{-i \delta} \\
2 \kappa^{2} v_{S}^{2} e^{-2 i \delta}+2 A_{\kappa} \kappa v_{S} e^{i \delta} & m_{\sigma}^{2}
\end{array}\right],
\end{aligned}
$$

where

$$
\begin{aligned}
& M_{1}^{2}=M^{2}+4 \lambda_{N}^{2} v_{S}^{2}, \\
& m_{\sigma}^{2}=m_{S}^{2}+4 \kappa^{2} v_{S}^{2} .
\end{aligned}
$$

If $A_{\lambda}$ is real, the mass square eigenvalues of the sneutrino are:

$$
M_{ \pm}^{2}=M_{1}^{2} \pm \sqrt{A_{\lambda}^{2} \lambda_{N}^{2} v_{S}^{2}+\lambda_{N}^{2} \kappa^{2} v_{S}^{4}+2 A_{\lambda} \lambda_{N}^{2} v_{S}^{3} \kappa \cos (3 \delta)}
$$

which have the following eigenstates:

$$
\tilde{N}_{ \pm}=\frac{1}{\sqrt{2}}\left(\tilde{N} \pm \tilde{N}^{*}\right) .
$$

Similarly, one can write the mass square eigenvalues and eigenstates for the $\sigma-\sigma^{*}$ system. Because of mixing between the particle and anti-particle states of sneutrino and singlet scalar, these systems are similar to $K_{0}-\bar{K}_{0}$ and $B_{0}-\bar{B}_{0}$ systems [39]. The evolution of these systems in the non-relativistic limit are driven by the Hamiltonian $\mathcal{H}$ defined as follows:

$$
\mathcal{H}=\mathcal{M}-\frac{i}{2} \Gamma,
$$

where $\mathcal{M}$ is the mass matrix and $\Gamma$ is the decay rate matrix of the corresponding system. 
Finally, the decay rates of the time evolved particle and anti-particle states of the $\tilde{N}-\tilde{N}^{*}$ and the $\sigma-\sigma^{*}$ system are calculated to get the final total CP asymmetry. As can be seen from eq. (2.11), both $\tilde{N}$ and $\sigma$ can decay to produce lepton asymmetry depending on the nature of their couplings. Therefore, we separately consider the two limiting cases where only one of them can decay at a time by fixing their masses. The first case is when $M_{1} \gg m_{\sigma}$. In this case, $\tilde{N}$ decays to produce the CP asymmetry at the tree-level while decays of $\sigma$ into a pair of $\mathrm{RH}$ (s)neutrinos are kinematically suppressed. The relevant Feynman diagrams for this situation are shown in figures 1 and 2. Figure 1 shows the point interaction, 2 and 3 body decays of $\tilde{N}$ while figure 2 shows the other possible 3 -body decays of $\tilde{N}$ that are mediated by an off-shell $\tilde{N}$. The first three point interactions of figure 1 are present in MSSM soft-leptogenesis as well and the observed matter-anti-matter asymmetry requires the decay products to have thermal corrections. The last diagram of figure 1 and those in figure 2 are presnt only in NMSSM. We will see in section 3.1 that the 3-body decay diagram of figure 1 is responsible for creating enough CP asymmetry without any thermal mass corrections. The contribution of diagrams in figure 2 is suppressed by the higher order soft terms. The second possibility to create the $\mathrm{CP}$ asymmetry is through decays of $\sigma$ which occurs when $m_{\sigma} \gg M_{1}$. The diagrams are shown in figure 3 . However, as we will see in section 3.2, these decays can not produce any CP asymmetry at the tree-level via the same mechanism followed by $\tilde{N}$ decays. This tells us that we can ignore the decays of $\sigma$ without any loss of generality for the study of soft leptogenesis in the present setup. We consider these cases one-by-one.

\section{$3.1 \quad \tilde{N}$ decays}

Irrespective of the mass of $\sigma$ relative to the mass of $\tilde{N}$, the sneutrino can decay into leptonic (sleptonic) and Higgs (Higgsino) final particles. The CP asymmetry generated from such a scenario was calculated in $[11,12]$. However, if $M_{1} \gg m_{\sigma}$, the 3-body decay channel shown in the last Feynman diagram of figure 1 opens up and it leads to interesting consequences for soft leptogenesis as we show below.

For the snuetrino system, upto leading order in the off-diagonal terms, ${ }^{2}$ the mass matrix can be calculated from the squared mass matrix in eq. (3.1):

$$
\mathcal{M}_{\tilde{N}}=M_{1}\left[\begin{array}{cc}
1 & \frac{\lambda_{N} \kappa v_{S}^{2} e^{2 i \delta}}{2 M_{1}^{2}}+\frac{A_{\lambda} \lambda_{N} v_{S} e^{-i \delta}}{2 M_{1}^{2}} \\
\frac{\lambda_{N} \kappa v_{S}^{2} e^{-2 i \delta}}{2 M_{1}^{2}}+\frac{A_{\lambda} \lambda_{N} v_{S} e^{i \delta}}{2 M_{1}^{2}} & 1
\end{array}\right] .
$$

The decay rate matrix can be written from the eq. (2.11). It contains both diagonal as well as off-diagonal terms because $\tilde{N}$ can decay into particle as well as anti-particle final states.

$$
\Gamma_{\tilde{N}}=\Gamma_{1}\left[\begin{array}{cc}
1+\alpha+\beta+\frac{4 \lambda_{N}^{2} v_{S}^{2}}{M_{1}^{2}}+\frac{\left|A_{N}\right|^{2}}{M_{1}^{2}} & \frac{4 \lambda_{N} v_{S} e^{-i \delta} A_{N}^{*}}{M_{1}^{2}} \\
\frac{4 \lambda_{N} v_{S} e^{i \delta} A_{N}}{M_{1}^{2}} & 1+\alpha+\beta+\frac{4 \lambda_{N}^{2} v_{S}^{2}}{M_{1}^{2}}+\frac{\left|A_{N}\right|^{2}}{M_{1}^{2}}
\end{array}\right],
$$

with,

$$
\Gamma_{1}=\frac{\left|Y_{N}\right|^{2} M_{1}}{8 \pi} .
$$

\footnotetext{
${ }^{2}$ Considering $\kappa$ and $A_{\lambda}$ much smaller compared to $\lambda_{N}$ and $M_{1}$ respectively as will be justified later.
} 


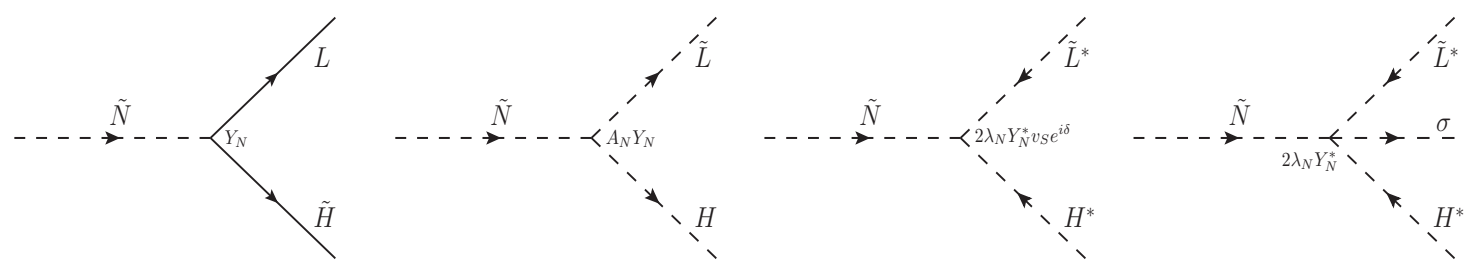

Figure 1. 2-body and 3-body decay diagrams of a singlet sneutrino, $\tilde{N}$.
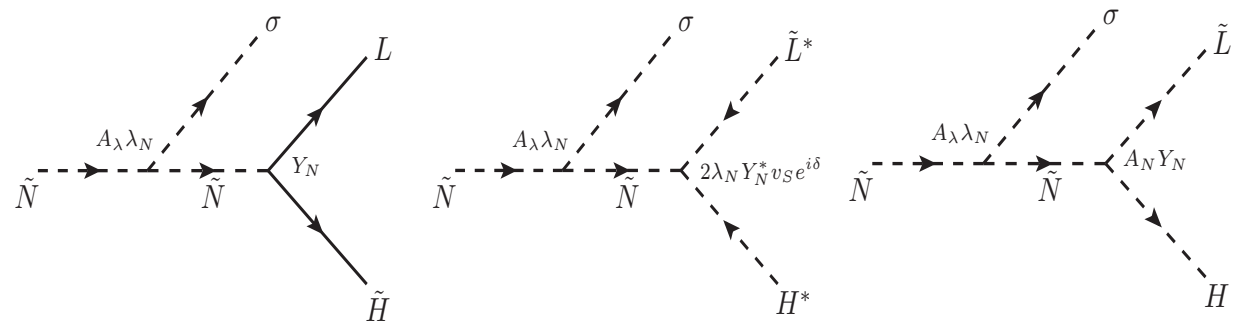

Figure 2. 3-body decay diagrams of singlet sneutrino, $\tilde{N}$ at higher order.

The parameter $\alpha$ and $\beta$ in $\Gamma_{\tilde{N}}$ matrix are associated with the 3 -body decay contributions of $\tilde{N}$ given by,

$$
\begin{aligned}
\alpha \simeq & \frac{\lambda_{N}^{2}}{\pi^{2}}\left[\frac{M_{1}\left(M_{1}^{2}+4 m_{\sigma}^{2}\right)^{1 / 2}}{2 M_{1}^{2}}-2 \frac{m_{\sigma}^{2}}{M_{1}^{2}} \log \left\{\frac{\left(M_{1}^{2}+4 m_{\sigma}^{2}\right)^{1 / 2}+M_{1}}{2 m_{\sigma}}\right\}+\frac{\left|A_{\lambda}\right|^{2} v_{S}^{2}}{2 M_{1}^{4}} \log \left(\frac{m_{N}}{2 m_{\sigma}}\right)\right. \\
& \left.+\frac{A_{\lambda} v_{S} \cos \delta}{2 M_{1}^{2}}\left\{-1+\frac{m_{\sigma}}{2 M_{1}}+\frac{m_{\sigma}^{2}}{4 M_{1}^{2}} \log \left(\frac{2 M_{1} m_{\sigma}-m_{\sigma}^{2}}{M_{1}^{2}-m_{\sigma}^{2}}\right)\right\}\right], \\
\beta \simeq & \frac{\lambda_{N}^{2}\left|A_{\lambda}\right|^{2}}{2 \pi^{2} M_{1}^{2}}\left[\log \left\{\frac{1}{8}+\frac{\left|A_{N}\right|^{2}}{M_{1}^{2}}\right\}-\frac{1}{8}\right] .
\end{aligned}
$$

The logarithm in $\beta$ can be expanded for the soft term $A_{N}$ at least an order of magnitude smaller than $M_{1}$ (this can be assumed without any loss of generality as later to draw our conclusions and compare with MSSM soft-leptogenesis in [11-13], we will be restricting to leading order in soft-terms) to give:

$$
\beta \simeq \frac{\lambda_{N}^{2}\left|A_{\lambda}\right|^{2}}{2 \pi^{2} M_{1}^{2}}\left[\frac{A_{N}^{2}}{M_{1}^{2}}-\frac{3}{4}\right]
$$

Under the same approximation used for $\beta$ and with $M_{1} \gg m_{\sigma}, \alpha$ becomes:

$$
\alpha \simeq \frac{\lambda_{N}^{2}}{\pi^{2}}\left[\frac{1}{2}-\frac{A_{\lambda} v_{S} \cos \delta}{2 M_{1}^{2}}\right]
$$

It is important to note that as long as the soft term $A_{\lambda}$ is sufficiently small compared to $M_{1}$ (at least by an order of magnitude) or the phase $\pi / 2<\delta<3 \pi / 2, \alpha \neq 0$. 
There are 4 terms in $\alpha$ of eq. (3.10) which represents the contribution of $\tilde{N} \rightarrow \sigma \tilde{L}^{*} H^{*}$ decay, where the first two terms come from the 3-body decay arising from the point interaction in figure 1 while the third term comes from the second diagram of figure 2 . The last term in $\alpha$ comes from the interference of these two diagrams. The $\beta$ in eq. (3.11) appears in the decay matrix $\Gamma_{\tilde{N}}$ due to the presence of first and the third diagrams of figure 2 . It is pertinent to note here that $\beta$ is at least quadratic in the soft terms $A_{\lambda}$ and $A_{N}$. One could in principle have higher order diagrams relating to 4 or more decay products. However, all such diagrams will be suppressed heavily by higher powers of $A_{\lambda} / M_{1}$.

The solutions for the time evolution of $\tilde{N}$ and $\tilde{N}^{*}$ come from the Schrodinger like equation

$$
\mathcal{H} \psi=i \frac{d \psi}{d t},
$$

where $\psi=\left\{\tilde{N}, \tilde{N}^{*}\right\}^{T}$. The solutions are obtained as,

$$
\begin{aligned}
\tilde{N}(t) & =e^{-i a t}\left[\tilde{N}_{0} \cos \left(\frac{p}{q} b t\right)-i \tilde{N}_{0}^{*} \frac{q}{p} \sin \left(\frac{p}{q} b t\right)\right], \\
\tilde{N}^{*}(t) & =e^{-i a t}\left[\tilde{N}_{0}^{*} \cos \left(\frac{p}{q} b t\right)-i \tilde{N}_{0} \frac{p}{q} \sin \left(\frac{p}{q} b t\right)\right],
\end{aligned}
$$

where $\tilde{N}_{0}, \tilde{N}_{0}^{*}$ are the field values at $t=0$ and

$$
\begin{aligned}
a & =\left(\mathcal{M}_{\tilde{N}}\right)_{11}-i \frac{\left(\Gamma_{\tilde{N}}\right)_{11}}{2}=\left(\mathcal{M}_{\tilde{N}}\right)_{22}-i \frac{\left(\Gamma_{\tilde{N}}\right)_{22}}{2}, \\
b & =\left(\mathcal{M}_{\tilde{N}}\right)_{12}-i \frac{\left(\Gamma_{\tilde{N}}\right)_{12}}{2}, \\
\left(\frac{p}{q}\right)^{2} & =\frac{\left(\mathcal{M}_{\tilde{N}}\right)_{12}^{*}-i \frac{\left(\Gamma_{\tilde{N}}\right)_{12}^{*}}{2}}{\left(\mathcal{M}_{\tilde{N}}\right)_{12}-i \frac{\left(\Gamma_{\tilde{N}}\right)_{12}}{2}} .
\end{aligned}
$$

Let's define $\Delta M=M_{+}-M_{-}$and $\Delta \Gamma_{\tilde{N}}=\Gamma_{+}-\Gamma_{-}$and $Q=\frac{p}{q} b$. Then if $\Gamma_{1}^{2} \ll M_{1}^{2}$ which happens when $Y_{N} \ll 1$ (typically of $\mathcal{O}\left(10^{-4}\right)$ to satisfy neutrino mass bounds), we can write

$$
\begin{aligned}
2 \operatorname{Re}(Q) & \simeq \Delta M=\frac{\lambda_{N} v_{S}}{M_{1}}\left[A_{\lambda}^{2}+\kappa^{2} v_{S}^{2}+2 A_{\lambda} v_{S} \kappa \cos (3 \delta)\right]^{1 / 2}, \\
-4 \operatorname{Im}(Q) & \simeq \Delta \Gamma_{\tilde{N}}=\frac{2 Y_{N}^{2} \lambda_{N}^{2} v_{S}^{2}}{\pi^{2} M_{1}^{2} \Delta M}\left\{\kappa v_{S}\left(\cos (3 \delta) \operatorname{Im} A_{N}+\sin (3 \delta) \operatorname{Re} A_{N}\right)+A_{\lambda} \operatorname{Re} A_{N}\right\} .
\end{aligned}
$$

If $\Delta \Gamma_{\tilde{N}} \ll \Delta M$ as well, the argument of the trigonometric functions becomes $\Delta M t / 2$ such that we can write:

$$
\begin{aligned}
\tilde{N}(t) & =g_{1} \tilde{N}_{0}+\frac{q}{p} g_{2} \tilde{N}_{0}^{*}, \\
\tilde{N}^{*}(t) & =g_{1} \tilde{N}_{0}^{*}+\frac{p}{q} g_{2} \tilde{N}_{0},
\end{aligned}
$$

where

$$
\begin{aligned}
& g_{1}=e^{-i M_{1} t} \exp \left[-\frac{\Gamma_{1}}{2}\left(1+\alpha+\beta+\frac{4 \lambda_{N}^{2} v_{S}^{2}}{M_{1}^{2}}+\frac{\left|A_{N}\right|^{2}}{M_{1}^{2}}\right) t\right] \cos \left[\frac{\Delta M t}{2}\right], \\
& g_{2}=-i e^{-i M_{1} t} \exp \left[-\frac{\Gamma_{1}}{2}\left(1+\alpha+\beta+\frac{4 \lambda_{N}^{2} v_{S}^{2}}{M_{1}^{2}}+\frac{\left|A_{N}\right|^{2}}{M_{1}^{2}}\right) t\right] \sin \left[\frac{\Delta M t}{2}\right] .
\end{aligned}
$$


The eqs. (3.22) and (3.23) are substituted back in eq. (2.11) for calculating the CP asymmetry factor $\varepsilon$ which is defined as the ratio of the difference between the decay rates of $\tilde{N}$ and $\tilde{N}^{*}$ into final state particles with lepton number +1 and -1 to the sum of all the decay rates, i.e.,

$$
\varepsilon=\frac{\sum_{f} \int_{0}^{\infty} d t\left[\Gamma(\tilde{N}(t) \rightarrow f)+\Gamma\left(\tilde{N}^{*}(t) \rightarrow f\right)-\Gamma(\tilde{N}(t) \rightarrow \bar{f})-\Gamma(\tilde{N}(t) \rightarrow \bar{f})\right]}{\sum_{f} \int_{0}^{\infty} d t\left[\Gamma(\tilde{N}(t) \rightarrow f)+\Gamma\left(\tilde{N}^{*}(t) \rightarrow f\right)+\Gamma(\tilde{N}(t) \rightarrow \bar{f})+\Gamma\left(\tilde{N}^{*}(t) \rightarrow \bar{f}\right)\right]},
$$

where $f, \bar{f}$ are the final states with lepton number +1 and -1 , respectively. This then gives us the following $\mathrm{CP}$ asymmetry parameter:

$$
\varepsilon=\frac{\int_{0}^{\infty} d t \frac{\left|g_{2}\right|^{2}\left|Y_{N}\right|^{2} M_{1}}{8 \pi}\left(\left|\frac{q}{p}\right|^{2}-\left|\frac{p}{q}\right|^{2}\right)\left[1+\frac{\left|A_{N}\right|^{2}}{M_{1}^{2}}-\frac{4 \lambda_{N}^{2} v_{S}^{2}}{M_{1}^{2}}-\alpha+\beta\right]}{\int_{0}^{\infty} d t \frac{\left|Y_{N}\right|^{2} M_{1}}{4 \pi}\left(1+\frac{\left|A_{N}\right|^{2}}{M_{1}^{2}}+\frac{4 \lambda_{N}^{2} v_{S}^{2}}{M_{1}^{2}}+\alpha+\beta\right)\left[\left|g_{1}\right|^{2}+\frac{\left|g_{2}\right|^{2}}{2}\left(\left|\frac{q}{p}\right|^{2}+\left|\frac{p}{q}\right|^{2}\right)\right]} .
$$

The decay rates at the tree-level itself for final states with lepton numbers \pm 1 are different because the factor $\left|\frac{p}{q}\right| \neq 1$ as it is not a hermitian quantity. This requires non-zero off-diagonal terms to be present in the mass matrix as well as the decay rate matrix of the system with atleast one of them being complex.

The sum and difference of the ratios $|q / p|^{2}$ and $|p / q|^{2}$ can be written as:

$$
\begin{aligned}
& \left|\frac{q}{p}\right|^{2}-\left|\frac{p}{q}\right|^{2}=-2\left(\frac{y^{2}}{x^{2}-y^{2}}\right)^{1 / 2} \\
& \left|\frac{q}{p}\right|^{2}+\left|\frac{p}{q}\right|^{2}=2\left(\frac{x^{2}}{x^{2}-y^{2}}\right)^{1 / 2}
\end{aligned}
$$

where

$$
\begin{aligned}
& x=\frac{\lambda_{N}^{2} \kappa^{2} v_{S}^{4}}{4}+\frac{A_{\lambda}^{2} \lambda_{N}^{2} v_{S}^{2}}{4}+\frac{4 \Gamma_{1}^{2} \lambda_{N}^{2} v_{S}^{2}\left|A_{N}\right|^{2}}{M_{1}^{2}}+\frac{\lambda_{N}^{2} A_{\lambda} \kappa v_{S}^{3} \cos (3 \delta)}{2}, \\
& y=\frac{2 \Gamma_{1} A_{\lambda} \lambda_{N}^{2} v_{S}^{2} \operatorname{Im} A_{N}}{M_{1}}+\frac{4 \lambda_{N}^{2} \kappa v_{S}^{3} \Gamma_{1}}{M_{1}}\left(\cos (3 \delta) \operatorname{Im} A_{N}+\sin (3 \delta) \operatorname{Re} A_{N}\right) .
\end{aligned}
$$

Keeping terms upto the leading order in $\Gamma_{1}$, we find that the sum of the ratios is $\simeq 2$ while the difference is twice the values of $y /\left(x^{2}-y^{2}\right)^{1 / 2}$ with,

$$
\left(\frac{y^{2}}{x^{2}-y^{2}}\right)^{1 / 2}=\frac{\left|Y_{N}\right|^{2}\left[A_{\lambda} \operatorname{Im} A_{N}+\kappa v_{S}\left\{\cos (3 \delta) \operatorname{Im} A_{N}+\sin (3 \delta) \operatorname{Re} A_{N}\right\}\right]}{\pi\left[\kappa^{2} v_{S}^{2}+A_{\lambda}^{2}+2 A_{\lambda} \kappa v_{S} \cos (3 \delta)\right]}
$$

such that the final CP asymmetry can be written as:

$$
\varepsilon=-\frac{\Delta M^{2}}{2\left(\Gamma^{2}+\Delta M^{2}\right)} \frac{\left[1+\frac{\left|A_{N}\right|^{2}}{M_{1}^{2}}-\frac{4 \lambda_{N}^{2} v_{S}^{2}}{M_{1}^{2}}-\alpha+\beta\right]}{\left[1+\frac{\left|A_{N}\right|^{2}}{M_{1}^{2}}+\frac{4 \lambda_{N}^{2} v_{S}^{2}}{M_{1}^{2}}+\alpha+\beta\right]}\left(\frac{y^{2}}{x^{2}-y^{2}}\right)^{1 / 2},
$$


where $\Gamma$ is defined as $\Gamma=\Gamma_{1}\left[1+\frac{\left|A_{N}\right|^{2}}{M_{1}^{2}}+\frac{4 \lambda_{N}^{2} v_{S}^{2}}{M_{1}^{2}}+\alpha+\beta\right]$. From eqs. (3.32) and (3.33) it is evident that there is a non-zero $\mathrm{CP}$ asymmetry even with real $A_{N}$, provided $\delta$ is sufficiently large.

We also note that while the usual soft leptogenesis done in the MSSM [11, 12], to the leading order in soft terms $\left(M^{2} \ll M_{1}^{2}, A_{N}^{2} \ll M_{1}^{2}\right)$, necessarily requires thermal phase space factors for the final state bosons $\left(c_{B}\right)$ and fermions $\left(c_{F}\right)$ to have $\varepsilon \propto \Delta_{B F}=\frac{c_{B}-c_{F}}{c_{B}+c_{F}}$, we get an asymmetry even without thermal mass corrections to the decay products at the leading order in soft terms. This happens because of the presence of $\sigma$ in the model which facilitates a 3-body decay of $\tilde{N}$ which is not cancelled by the other terms. If we did not have this, at leading order in soft terms, $1-\frac{4 \lambda_{N}^{2} v_{S}^{2}}{M_{1}^{2}}=0$ and there would be no asymmetry without thermal mass corrections. At the leading order in the soft terms $\left(A_{\lambda} \ll M_{1}^{2} \Rightarrow \beta\right.$ is negligible), $\varepsilon$ is

$$
\varepsilon=\frac{\Delta M^{2}}{2\left(\Gamma^{2}+\Delta M^{2}\right)} \frac{\alpha}{(2+\alpha)}\left(\frac{y^{2}}{x^{2}-y^{2}}\right)^{1 / 2} .
$$

It is clear from eq. (3.34) that the only contribution coming from diagrams of figure 2 are coming from the cross term of the second diagram with the last diagram of figure 1 through $\alpha$. Even in $\alpha$, the dominant contribution comes through the 3-body decay by the vertex interaction of figure 1. Therefore it is possible to successfully generate non-zero lepton asymmetry from $\tilde{N}-\tilde{N}^{*}$ system at the tree-level without using thermal phase space factors for bosonic and fermionic final states. We discuss this more and give some numerical estimates in section 5 for relevant parameters. For the moment, let's consider the decays of $\sigma$.

\section{$3.2 \sigma$ decays}

Unlike the $\tilde{N}$ decays where most of the decay products were massless, the final products of $\sigma$ decay are massive, as shown in figure 3. This creates two possible decay modes of $\sigma$ according to the condition satisfied.

1. $\sigma$ decays to $N N$ and $\tilde{N} \tilde{N}$. This happens when $m_{\sigma}^{2}>4 M_{1}^{2}$,

2. $\sigma$ decays only to $N N$. This happens when $16 \lambda_{N}^{2} v_{S}^{2}=4 m_{N}^{2}<m_{\sigma}^{2}<4 M_{1}^{2}$.

The mass matrix and the $\Gamma$ matrix of the $\sigma-\sigma^{*}$ system are respectively:

$$
M_{\sigma}=m_{\sigma}\left[\begin{array}{cc}
1 & \frac{\kappa^{2} v_{S}^{2} e^{2 i \delta}+A_{\kappa}^{*} \kappa v_{S} e^{-i \delta}}{m_{\sigma}^{2}} \\
\frac{\kappa^{2} v_{S}^{2} e^{-2 i \delta}+A_{\kappa} \kappa v_{S} e^{i \delta}}{m_{\sigma}^{2}} & 1
\end{array}\right], \quad \Gamma_{\sigma}=\Gamma_{\sigma, 1}\left[\begin{array}{cc}
\Theta & 0 \\
0 & \Theta
\end{array}\right],
$$

where

$$
\begin{aligned}
\Gamma_{\sigma, 1} & =\frac{\lambda_{N}^{2}}{32 \pi m_{\sigma}^{2}}, \\
\Theta & =\left\{\begin{array}{cc}
\left(m_{\sigma}^{2}-4 m_{N}^{2}\right)^{3 / 2}+A_{\lambda}^{2}\left(m_{\sigma}^{2}-4 M_{1}^{2}\right)^{1 / 2} & \text { for case } 1, \\
\left(m_{\sigma}^{2}-4 m_{N}^{2}\right)^{3 / 2} & \text { for case } 2 .
\end{array}\right.
\end{aligned}
$$




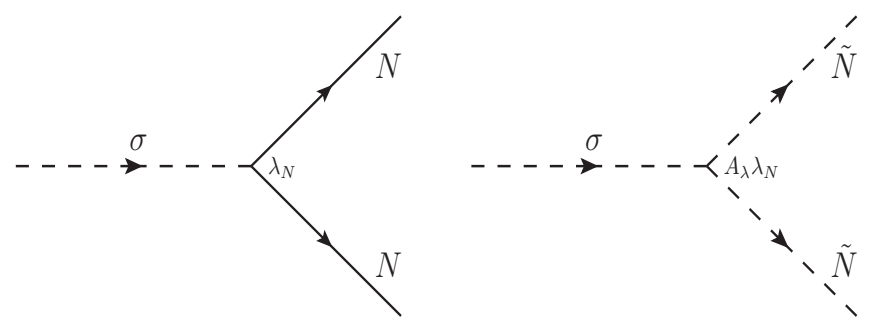

Figure 3. Decay diagrams of a singlet scalar $\sigma$.

A non-relativistic Hamiltonian can be defined following eq. (3.6). Immediately it can be seen that because of the absence of an off-diagonal term in the decay rate matrix of $\sigma$, the ratio corresponding to $(p / q)^{2}$ of $\tilde{N}$ decay,

$$
\left(\frac{s}{r}\right)^{2}=\frac{\left(M_{\sigma}\right)_{12}^{*}-i \frac{\left(\Gamma_{\sigma}^{*}\right)_{12}}{2}}{\left(M_{\sigma}\right)_{12}-i \frac{\left(\Gamma_{\sigma}\right)_{12}}{2}}=\frac{\left(M_{\sigma}\right)_{12}^{*}}{\left(M_{\sigma}\right)_{12}} .
$$

If we solve for the evolution of the $\sigma-\sigma^{*}$ system, the CP asymmetry parameter computed exactly analogously to the $\tilde{N}-\tilde{N}^{*}$ system will be zero because of exact cancellation between the ratios $|r / s|^{2}$ and $|s / r|^{2}$. Thus

$$
\varepsilon_{\sigma}=0
$$

\section{General constraints}

The CP asymmetry in this model depends on a lot of parameters. However, we can constrain some of them by various considerations. In deriving the following general constraints, we take $M_{1} \gg M \Rightarrow M_{1} \simeq 2 \lambda_{N} v_{S}, M_{1} \gg m_{\sigma}$ and $A_{\lambda} \simeq \kappa v_{S}$ for reasons that will become clear later in section 5 .

- The condition of out-of-equilibrium decay at $T=M_{1}$ is given by comparing the decay rate of the $\tilde{N}$ with the Hubble parameter at $T=M_{1}$ :

$$
\Gamma \lesssim H\left(T=M_{1}\right)=\sqrt{\frac{8 \pi^{3} g_{s}}{90}} \frac{M_{1}^{2}}{m_{P l}},
$$

where $\Gamma$ is the diagonal component of eq. (3.8). Substituting it in eq. (4.1) and neglecting the contribution of $\beta$ we get

$$
\frac{\left|A_{N}\right|^{2}}{M_{1}^{2}} \lesssim \frac{13 \pi \sqrt{g_{*}} M_{1}}{Y_{N}^{2} m_{P l}}-2-\alpha .
$$

For $M_{1} \gg m_{\sigma}, \alpha \approx \mathcal{O}\left(10^{-2}\right)$ and we may write eq. (4.2) as

$$
\frac{\left|A_{N}\right|^{2}}{M_{1}^{2}} \lesssim \frac{13 \pi \sqrt{g_{*}} M_{1}}{Y_{N}^{2} m_{P l}}-2
$$


- The way we derived the $\mathrm{CP}$ asymmetry requires well separated states [12], i.e., $\Gamma \ll \Delta M$ as well as $\Delta \Gamma \ll \Delta M$ as stated before. This gives us two self-consistent limits:

$$
\begin{aligned}
\frac{\left|A_{N}\right|^{2}}{M_{1}^{2}} & \ll \frac{8 \pi \kappa v_{S}}{Y_{N}^{2} M_{1}}-2, \\
\cos (3 \delta) \operatorname{Im} A_{N}+(\sin (3 \delta)+1) \operatorname{Re} A_{N} & \ll \frac{2 \pi^{2}}{Y_{N}^{2}} \kappa v_{S} .
\end{aligned}
$$

- Neutrino mass upper limits $\left(m_{\nu} \lesssim 0.1 \mathrm{eV}[1,40]\right)$ put constraints on the Yukawa coupling strength $Y_{N}$ and the mass of the $\mathrm{RH}$ (s)neutrino.

$$
\frac{Y_{N}^{2}}{\lambda_{N} v_{S}} \lesssim 6.6 \times 10^{-15} \mathrm{GeV}
$$

- Electric dipole moment (EDM) calculations can constrain the $\mathrm{CP}$ violating phases that appear in the vevs of the two Higgs doublets and the scalar singlet $S$. In ref. [41], they show that in principle $\delta_{u}$ (the phase in the vev of $H_{u}$ should we go below the EW scale) and $\delta$ could be large as long as the relative phase is kept small. For more details about the EDM constraints on the NMSSM, see [42].

\subsection{A simpler form for $\varepsilon$}

We can write a simpler form for the CP asymmetry by using the approximations made and the general relationships between various parameters given above. The set of parameters governing $\varepsilon$ are:

$$
\left\{m_{S}, M, \kappa, \lambda_{N}, v_{S}, Y_{N}, A_{\lambda}, \operatorname{ReA}_{N}, \operatorname{Im} A_{N}, \delta\right\}
$$

We choose the soft masses $M, m_{S} \sim \mathcal{O}(1) \mathrm{TeV}$ and $v_{S}$ to be of $\mathcal{O}\left(10^{7}\right) \mathrm{GeV}$ with $\lambda_{N} \sim \mathcal{O}(1)$. This along with $\kappa \ll \lambda_{N}$ means both $M, m_{\sigma} \ll 2 \lambda_{N} v_{S} \simeq M_{1}$. Therefore, from eq. (4.6), one can put an upper limit on $Y_{N}$, i.e., $Y_{N} \lesssim \mathcal{O}\left(10^{-4}\right)$. With these choices and approximations the form of $\varepsilon$ can be simplified to

$$
\varepsilon \simeq \frac{1}{2 \pi}\left(\frac{\alpha}{2+\alpha}\right) \times \frac{Y_{N}^{2}\left[A_{\lambda} \operatorname{Im} A_{N}+\kappa v_{S}\left\{\cos (3 \delta) \operatorname{Im} A_{N}+\sin (3 \delta) \operatorname{Re} A_{N}\right\}\right]}{A_{\lambda}^{2}+\kappa^{2} v_{S}^{2}+2 A_{\lambda} \kappa v_{S} \cos (3 \delta)} .
$$

\section{$5 \quad$ Results and discussions}

To obtain the baryon asymmetry of the Universe, $\eta_{B}$, we solve the simultaneous Boltzmann equations for the $\tilde{N}$ number density, $N_{\tilde{N}}$, and the $B-L$ number density, $N_{B-L}$, which are as follows [43-45]:

$$
\begin{aligned}
\frac{d N_{\tilde{N}}}{d z} & =-K_{\tilde{N}} z\left(N_{\tilde{N}}-N_{\text {eq }}\right) \frac{\kappa_{1}(z)}{\kappa_{2}(z)}, \\
\frac{d N_{B-L}}{d z} & =-\varepsilon K_{\tilde{N}} z\left(N_{\tilde{N}}-N_{\text {eq }}\right) \frac{\kappa_{1}(z)}{\kappa_{2}(z)}-\frac{1}{4} K_{\tilde{N}} z^{3} \kappa_{1}(z) N_{B-L},
\end{aligned}
$$




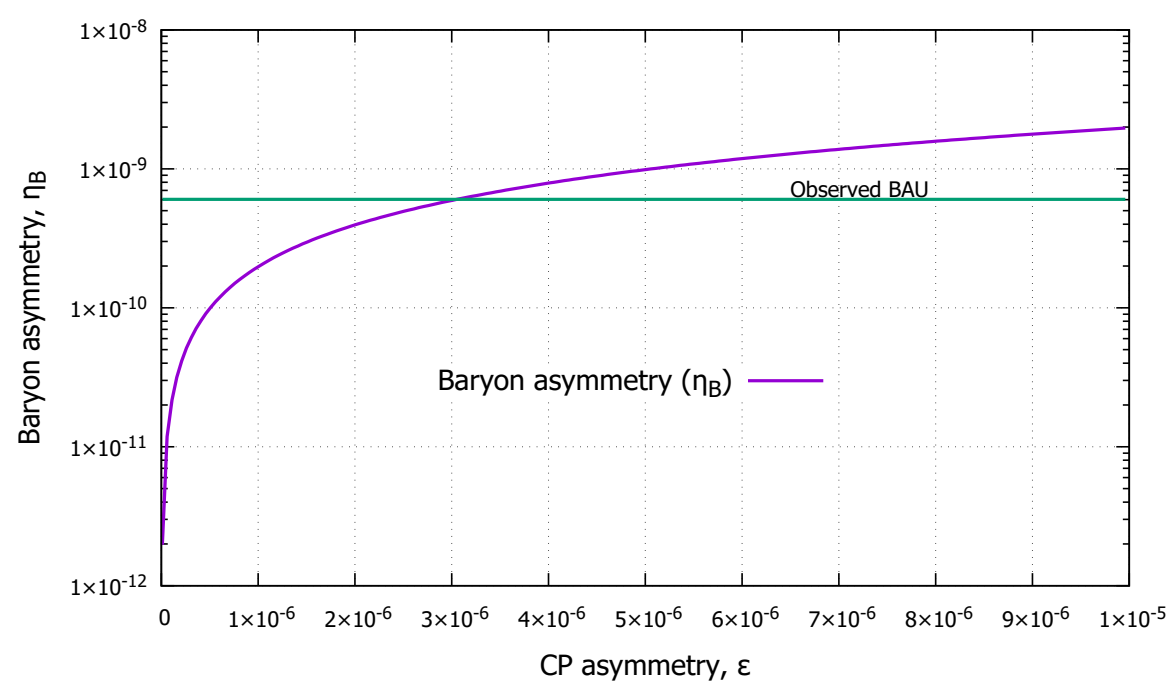

Figure 4. The dependence of the baryon asymmetry on the $\mathrm{CP}$ asymmetry. In this figure, we keep $Y_{N}=10^{-4.5}, \lambda_{N}=0.9, M=10^{4} \mathrm{GeV}, v_{S}=10^{6.5} \mathrm{GeV},\left|A_{N}\right|=10^{6.5} \mathrm{GeV}, m_{\sigma}=10^{2} \mathrm{GeV}$. The variation in $\varepsilon$ is brought on by not fixing $A_{\lambda}, \kappa, \delta$. We also take equilibrium initial condition for $\tilde{N}$ abundance. Starting with zero initial equilibrium for $\tilde{N}$ does not change the result.

where $K_{\tilde{N}}=\frac{\Gamma}{H(z=1)}$ is the Hubble parameter at $z=1$ with $z=\frac{M_{1}}{T}$ and $N_{\text {eq }}$ is the equilibrium number density of $\tilde{N}$. They take the following forms:

$$
\begin{aligned}
H(z=1) & =\sqrt{\frac{8 \pi^{3} g_{s}}{90}} \frac{M_{1}^{2}}{m_{P l}}, \\
N_{\text {eq }} & =\kappa_{2}(z) \frac{z^{2}}{2},
\end{aligned}
$$

with $m_{P l}=1.22 \times 10^{19} \mathrm{GeV}$ being the Planck mass and $g_{s}$ is the number of relativistic degrees of freedom in the NMSSM which we take $\approx 225$ except for the $\tilde{N}$ which is nonrelativistic. In writing the Boltzmann equation for $B-L$ number density, we neglect the $\Delta L=2$ scattering processes for washout and assume it is dominated mostly by inverse decays. The contribution to washout from the scattering processes is small because we are in the weak washout regime with $K_{\tilde{N}} \lesssim 1$. The final $B-L$ number density thus created, $N_{B-L}^{f}$ then converts to the baryon asymmetry by the sphaleron processes such that the ratio of the baryon number density to the photon number density, $\eta_{B}$, is:

$$
\eta_{B}=\frac{3}{4} \frac{g_{*}^{0}}{g_{*}} a_{\mathrm{sph}} N_{B-L}^{f},
$$

where $g_{*} \simeq g_{s} \simeq 225, g_{*}^{0}$ is the effective number of relativistic degrees of freedom at recombination and $a_{\mathrm{sph}}$ is the sphaleron conversion factor. Since we will solve the Boltzmann equations numerically, we use the complete form of $\varepsilon$ given in eq. (3.33).

In figure 4, we show the typical value of the $\mathrm{CP}$ asymmetry that satisfies the observed baryon asymmetry of the Universe. It turns out that we need $\varepsilon \simeq \mathcal{O}\left(10^{-6}\right)$ to get the correct observed baryon asymmetry while satisfying neutrino mass bounds. This value of 

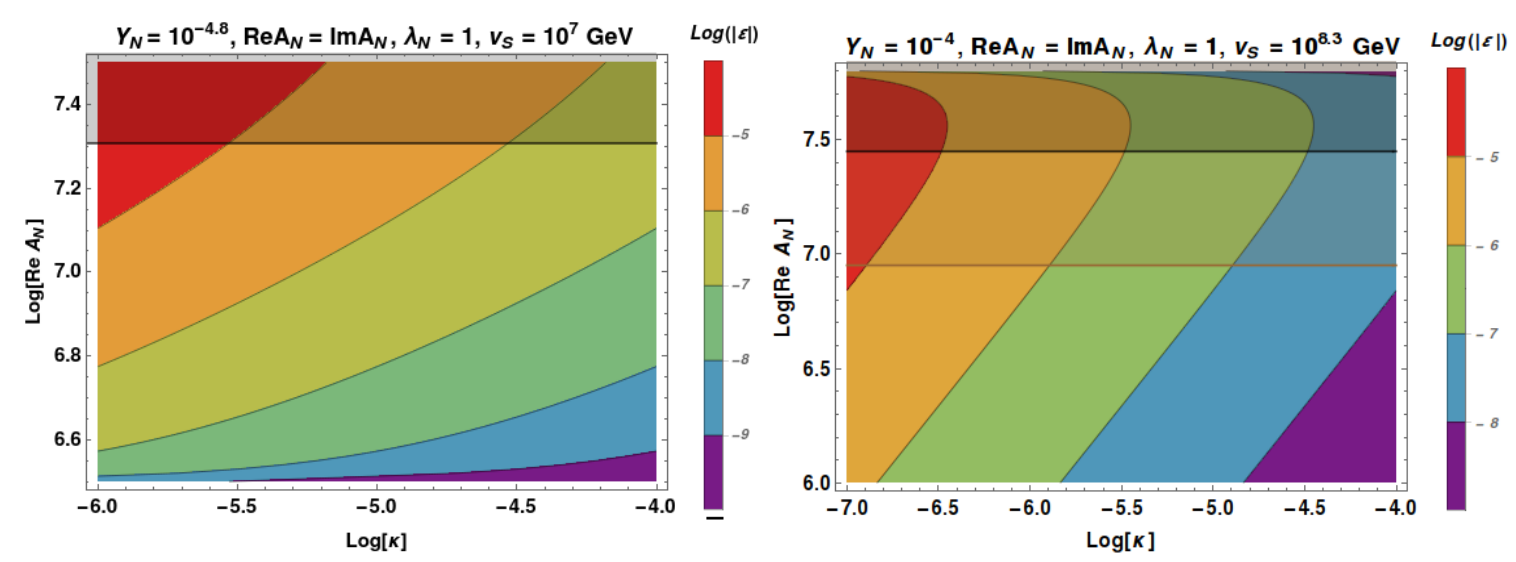

Figure 5. The contour plots of $\varepsilon$ in $\operatorname{Re} A_{N}-\kappa$ plane considering $A_{N}$ complex. The left panel shows only the out-of-equilibrium bound $(\Gamma \simeq H(z=1)$, in horizontal black solid line) with a generic $A_{N}$. In the right panel the horizontal brown solid line marks the leading order approximation in the soft-term $A_{N}\left(A_{N}^{2} / M_{1}^{2} \simeq 10^{-3}\right)$, while the black line is the out-of-equilibrium bound. All logarithms are to the base 10 .

$\varepsilon$ is similar to the one obtained by other vanilla leptogenesis scenarios in the weak washout regime. The only difference is that usual leptogenesis occurs with decays at the loop-level interfering with tree-level decays due to complex Yukawa couplings that violate CP. In the soft leptogenesis, the Yukawa parameter could very well remain real as the source of CP asymmetry lies elsewhere - in the time-varying mixing between $\tilde{N}$ and $\tilde{N}^{*}$ states due to the complex nature of $\langle S\rangle$ and the soft SUSY breaking parameters.

\subsection{Case 1: $\delta$ is near zero}

To get $\varepsilon \simeq \mathcal{O}\left(10^{-6}\right)$ we fix the values of the following parameters in line with the earlier approximations and constraints:

$$
\delta=0.3, M=1 \mathrm{TeV}, m_{S}=1 \mathrm{TeV}, \lambda_{N}=1 .
$$

For simplicity, we also assume $A_{\lambda} \simeq \kappa v_{S}$ (such that $\alpha \approx \frac{\lambda_{N}^{2}}{2 \pi}$ ) in finding the correct set of values for other parameters. Using these, we show the relation between $A_{N}$ and $\kappa$ for different values of $Y_{N}$ and $v_{S}$ (satisfying the SM neutrino mass bounds) in figures 5 and 6 as contour plots in $\log \varepsilon$.

In figure 5 we take a complex $A_{N}$ with $\operatorname{Im} A_{N}=\operatorname{Re} A_{N}$ and vary $\kappa$ and $\operatorname{Re} A_{N}$ for $Y_{N}=10^{-4.8}, v_{S}=10^{7} \mathrm{GeV}$ (left panel) and $Y_{N}=10^{-4}, v_{S}=10^{8.3} \mathrm{GeV}$ (right panel). As can be seen from the left figure, we need large $A_{N}\left(\gtrsim M_{1}\right)$ to satisfy correct order of $\varepsilon$ while ensuring that $\Gamma \lesssim H(z=1)$. However from the right panel, it is clear that sufficient $\mathrm{CP}$ asymmetry can be created even at leading order in $A_{N}$ without using thermal phase space factors.

We keep $A_{N}$ real in figure 6 and vary $\kappa$ and $\operatorname{Re} A_{N}$ for similar values of $Y_{N}$ and $v_{S}$ as before. It's clear that non-zero asymmetry can be created even with real $A_{N}$ as long as $\delta \neq n \pi(n \in \mathbb{Z})$. However compared to figure 5 , we find that the bounds on $A_{N}$ and $\kappa$ in 

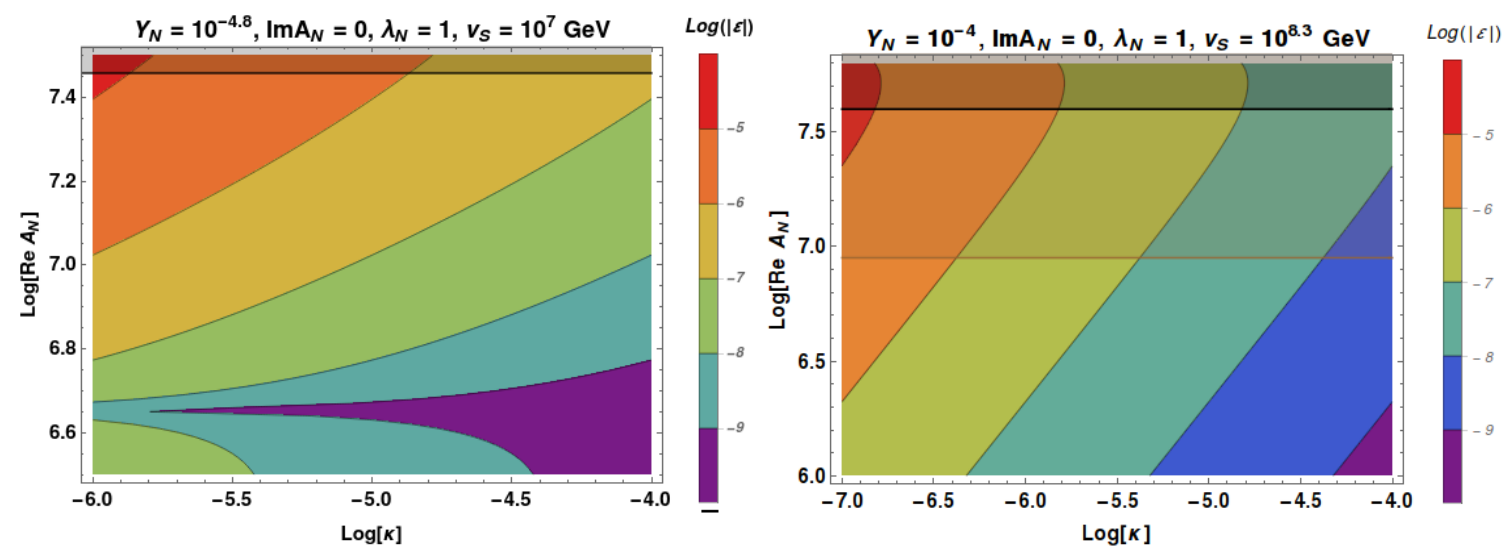

Figure 6. The contour plots of $\varepsilon$ in $\operatorname{Re} A_{N}-\kappa$ plane considering a real $A_{N}$. The left panel shows only the out-of-equilibrium bound $(\Gamma \simeq H(z=1)$, in horizontal black solid line) with a generic $A_{N}$. In the right panel the horizontal brown solid line marks the leading order approximation in the soft-term $A_{N}\left(A_{N}^{2} / M_{1}^{2} \simeq 10^{-3}\right)$, while the black line is the out-of-equilibrium bound. All logarithms are to the base 10 .

figure 6 are stronger as there is much less parameter space available satisfying $\varepsilon \gtrsim \mathcal{O}\left(10^{-6}\right)$. Both figures 5 and 6 satisfy neutrino mass bounds and the conditions of $\Gamma, \Delta \Gamma \ll \Delta M$.

\subsection{Case 2: $\delta=\pi$}

If the phase of the vev of $S$ is large, specially at $\delta=\pi$, we get a resonance behaviour in $\varepsilon$ at $A_{\lambda} \simeq \kappa v_{S}$. In the limit of $\delta \rightarrow \pi$, the CP asymmetry parameter of eq. (4.8) can be written as:

$$
\left.\varepsilon\right|_{\delta=\pi}=\frac{1}{2 \pi}\left(\frac{\alpha+\beta}{2+\alpha+\beta}\right) \frac{Y_{N}^{2} \operatorname{Im} A_{N}}{\left|A_{\lambda}-\kappa v_{S}\right|}
$$

Eq. (5.7) also justifies assuming $A_{\lambda} \simeq \kappa v_{S}$ to derive the general constraints on the various parameters in section 4 . The behaviour of $\varepsilon$ versus $A_{\lambda}$ is shown in figure 7 . For the plot, we take $M=m_{S}=1 \mathrm{TeV}$. The values of the other relevant parameters are shown in the figure itself. Since $\delta=\pi$, there is no contribution from the real part of $A_{N}$ in $\varepsilon$. This means that $A_{N}$ necessarily needs to be complex contrary to the case where $\delta$ is small. Without resonance, it was found in section 5.1 that $A_{N}$ needs to be several orders larger than $A_{\lambda}$ for correct amount of $\varepsilon \simeq \mathcal{O}\left(10^{-6}\right)$. However, the resonance effect at $\delta=\pi$ mitigates this requirement allowing $A_{N}$ to be of the same order or smaller than $A_{\lambda}$.

\section{Conclusion}

We have presented a new mechanism for soft leptogenesis in the context of the NMSSM with a singlet RH neutrino superfield. Similar to soft leptogenesis in the MSSM, we also generate $\mathrm{CP}$ asymmetry at the tree-level owing to the $\mathrm{CP}$ violation occuring due to the difference between the mass and CP eigenstates similar to the $K^{0}-\bar{K}^{0}$ or the $B^{0}-\bar{B}^{0}$ systems. The difference lies in the fact that MSSM soft leptogenesis requires using thermal 


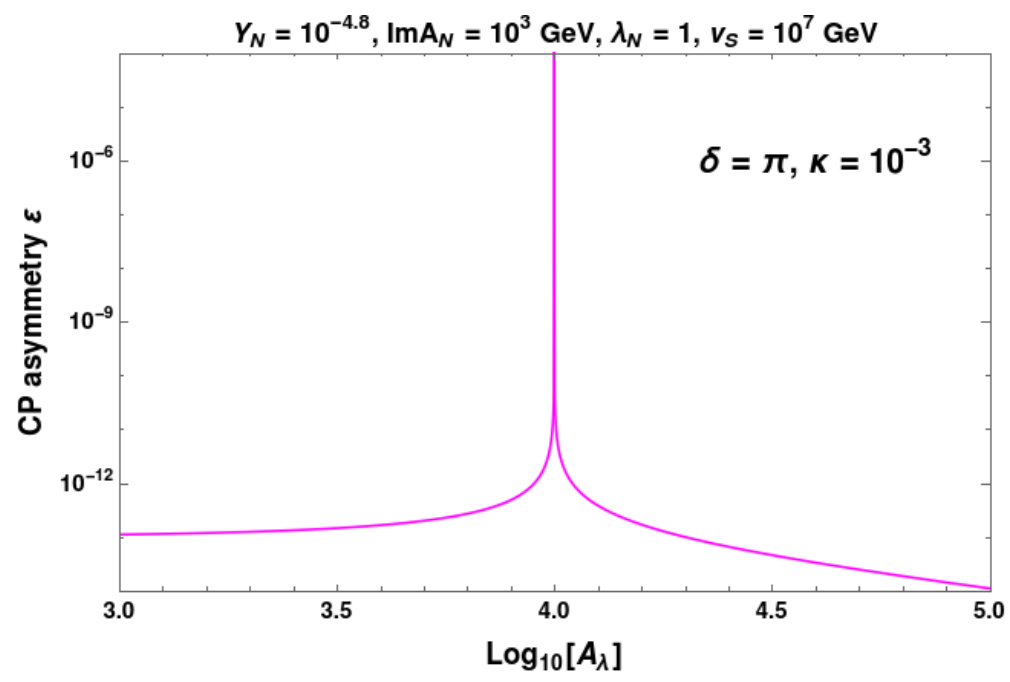

Figure 7. Variation of $\varepsilon$ versus $A_{\lambda}$ in the $\delta=\pi$ limit. The resonance in $\varepsilon$ occurs when $A_{\lambda}=\kappa v_{S}$.

masses and phase space factors for boson and fermion final states without which there is no asymmetry. In the NMSSM where the singlet scalar $S$ takes a vev, an asymmetry can be generated even without any thermal phase space/mass corrections to the decay products. Further if there is spontaneous CP violation in the system with $\sin \delta \neq 0$, lepton asymmetry can be created without using any other complex parameter. In the numerical analysis for small $\delta$ case, we considered the mass scale of the $\mathrm{RH}$ sneutrino to be $10^{7}-10^{8} \mathrm{GeV}$. We found that to generate sufficient asymmetry, one of the soft trilinear coupling $A_{N}$ needs to be $\gtrsim 10^{7} \mathrm{GeV}$ and $\kappa \lesssim \mathcal{O}\left(10^{-5}\right)$. This also tells us that $A_{\lambda} \simeq \mathcal{O}\left(10^{2}\right) \mathrm{GeV}$. However, if $\delta \rightarrow \pi$, there occurs a resonance in the system which helps to obtain $\epsilon \sim \mathcal{O}\left(10^{-6}\right)$ even with $A_{N} \lesssim A_{\lambda}$ and the value of $\kappa$ can be comparatively larger $\left(\gtrsim \mathcal{O}\left(10^{-3}\right)\right)$. The mass of the RH sneutrino came out in the range $\mathcal{O}\left(10^{7}-10^{8}\right) \mathrm{GeV}$ which lies below the cosmological gravitino overproduction bound of $T_{\text {reheat }} \simeq \mathcal{O}\left(10^{9}\right) \mathrm{GeV}$ [46-49]. This mass scale for the sneutrino (which depends on the vev of $S, v_{S}$ ) in the NMSSM also could favour gravitational wave detection at LIGO [50] provided a strong first order phase transition occurs in the scalar sector. It would be interesting to explore the flavor effects in the present scenario that we leave for a future study.

\section{Acknowledgments}

WA would like to acknowledge the support in the form of funding available from the Department of Atomic Energy, Government of India for the Neutrino Project at HarishChandra Research Institute (HRI). AK would like to thank Bharti Kindra for discussions on $K^{0}-\bar{K}^{0}$ systems. AK and AKS acknowledge PRL for providing postdoctoral research fellowship.

Open Access. This article is distributed under the terms of the Creative Commons Attribution License (CC-BY 4.0), which permits any use, distribution and reproduction in any medium, provided the original author(s) and source are credited. 


\section{References}

[1] Particle Data Group collaboration, Review of Particle Physics, Phys. Rev. D 98 (2018) 030001 [INSPIRE].

[2] M. Fukugita and T. Yanagida, Baryogenesis Without Grand Unification, Phys. Lett. B 174 (1986) 45 [INSPIRE].

[3] A. Strumia, Baryogenesis via leptogenesis, in Particle physics beyond the standard model. Proceedings, Summer School on Theoretical Physics, 84th Session, Les Houches, France, 1-26 August 2005, pp. 655-680 (2006) [hep-ph/0608347] [INSPIRE].

[4] P. Minkowski, $\mu \rightarrow e \gamma$ at a Rate of One Out of $10^{9}$ Muon Decays?, Phys. Lett. $67 \mathrm{~B}$ (1977) 421 [INSPIRE].

[5] R.N. Mohapatra and G. Senjanović, Neutrino Mass and Spontaneous Parity Nonconservation, Phys. Rev. Lett. 44 (1980) 912 [InSPIRE].

[6] T. Yanagida, Horizontal gauge symmetry and masses of neutrinos, Conf. Proc. C 7902131 (1979) 95 [INSPIRE].

[7] M. Gell-Mann, P. Ramond and R. Slansky, Complex Spinors and Unified Theories, Conf. Proc. C 790927 (1979) 315 [arXiv:1306.4669] [InSPIRE].

[8] S.L. Glashow, The Future of Elementary Particle Physics, NATO Sci. Ser. B 61 (1980) 687 [INSPIRE].

[9] J. Schechter and J.W.F. Valle, Neutrino Masses in $\mathrm{SU}(2) \times \mathrm{U}(1)$ Theories, Phys. Rev. D 22 (1980) 2227 [INSPIRE].

[10] V.A. Kuzmin, V.A. Rubakov and M.E. Shaposhnikov, On the Anomalous Electroweak Baryon Number Nonconservation in the Early Universe, Phys. Lett. 155B (1985) 36 [INSPIRE].

[11] Y. Grossman, T. Kashti, Y. Nir and E. Roulet, Leptogenesis from supersymmetry breaking, Phys. Rev. Lett. 91 (2003) 251801 [hep-ph/0307081] [InSPIRE].

[12] G. D'Ambrosio, G.F. Giudice and M. Raidal, Soft leptogenesis, Phys. Lett. B 575 (2003) 75 [hep-ph/0308031] [InSPIRE].

[13] C.S. Fong, M.C. Gonzalez-Garcia and E. Nardi, Leptogenesis from Soft Supersymmetry Breaking (Soft Leptogenesis), Int. J. Mod. Phys. A 26 (2011) 3491 [arXiv:1107.5312] [INSPIRE].

[14] M.-C. Chen and K.T. Mahanthappa, Lepton flavor violating decays, soft leptogenesis and SUSY SO(10), Phys. Rev. D 70 (2004) 113013 [hep-ph/0409096] [InSPIRE].

[15] E.J. Chun and S. Scopel, Soft leptogenesis in Higgs triplet model, Phys. Lett. B 636 (2006) 278 [hep-ph/0510170] [INSPIRE].

[16] A.D. Medina and C.E.M. Wagner, Soft leptogenesis in warped extra dimensions, JHEP 12 (2006) 037 [hep-ph/0609052] [INSPIRE].

[17] J. Garayoa, M.C. Gonzalez-Garcia and N. Rius, Soft leptogenesis in the inverse seesaw model, JHEP 02 (2007) 021 [hep-ph/0611311] [INSPIRE].

[18] C.S. Fong and M.C. Gonzalez-Garcia, Flavoured Soft Leptogenesis, JHEP 06 (2008) 076 [arXiv: 0804.4471] [INSPIRE]. 
[19] C.S. Fong and M.C. Gonzalez-Garcia, On Gaugino Contributions to Soft Leptogenesis, JHEP 03 (2009) 073 [arXiv: 0901.0008] [inSPIRE].

[20] Y. Kajiyama, S. Khalil and M. Raidal, Electron EDM and soft leptogenesis in supersymmetric B-L extension of the standard model, Nucl. Phys. B $\mathbf{8 2 0}$ (2009) 75 [arXiv: 0902.4405] [INSPIRE].

[21] C.S. Fong, M.C. Gonzalez-Garcia, E. Nardi and J. Racker, Flavoured soft leptogenesis and natural values of the B term, JHEP 07 (2010) 001 [arXiv: 1004.5125] [INSPIRE].

[22] K. Hamaguchi and N. Yokozaki, Soft Leptogenesis and Gravitino Dark Matter in Gauge Mediation, Phys. Lett. B 694 (2011) 398 [arXiv:1007.3323] [InSPIRE].

[23] C.S. Fong, M.C. Gonzalez-Garcia and E. Nardi, Early Universe effective theories: The Soft Leptogenesis and R-Genesis Cases, JCAP 02 (2011) 032 [arXiv: 1012.1597] [INSPIRE].

[24] H. Kuismanen and I. Vilja, Baryon asymmetry and dark matter from soft leptogenesis, Phys. Rev. D 87 (2013) 015005 [arXiv:1210.4335] [INSPIRE].

[25] D.J.H. Chung, L.L. Everett, G.L. Kane, S.F. King, J.D. Lykken and L.-T. Wang, The Soft supersymmetry breaking Lagrangian: Theory and applications, Phys. Rept. 407 (2005) 1 [hep-ph/0312378] [INSPIRE].

[26] S.P. Martin, A Supersymmetry primer, hep-ph/9709356 [INSPIRE].

[27] Y. Grossman, T. Kashti, Y. Nir and E. Roulet, New ways to soft leptogenesis, JHEP 11 (2004) 080 [hep-ph/0407063] [inSPIRE].

[28] R. Adhikari, A. Dasgupta, C.S. Fong and R. Rangarajan, Nonthermal CP violation in soft leptogenesis, Phys. Rev. D 91 (2015) 096001 [arXiv:1501.06310] [InSPIRE].

[29] J.E. Kim and H.P. Nilles, The mu Problem and the Strong CP Problem, Phys. Lett. 138B (1984) 150 [INSPIRE].

[30] U. Ellwanger, C. Hugonie and A.M. Teixeira, The Next-to-Minimal Supersymmetric Standard Model, Phys. Rept. 496 (2010) 1 [arXiv:0910.1785] [INSPIRE].

[31] M. Maniatis, The Next-to-Minimal Supersymmetric extension of the Standard Model reviewed, Int. J. Mod. Phys. A 25 (2010) 3505 [arXiv:0906. 0777] [inSPIRE].

[32] Y. Grossman and H.E. Haber, Sneutrino mixing phenomena, Phys. Rev. Lett. 78 (1997) 3438 [hep-ph/9702421] [INSPIRE].

[33] S.F. King, Atmospheric and solar neutrinos with a heavy singlet, Phys. Lett. B 439 (1998) 350 [hep-ph/9806440] [INSPIRE].

[34] R. Kitano and K.-y. Oda, Neutrino masses in the supersymmetric standard model with right-handed neutrinos and spontaneous R-parity violation, Phys. Rev. D 61 (2000) 113001 [hep-ph/9911327] [INSPIRE].

[35] D. Das and S. Roy, One-loop contribution to the neutrino mass matrix in NMSSM with right-handed neutrinos and tri-bimaximal mixing, Phys. Rev. D 82 (2010) 035002 [arXiv: 1003.4381] [INSPIRE].

[36] T. Alanne, T. Hugle, M. Platscher and K. Schmitz, Low-scale leptogenesis assisted by a real scalar singlet, JCAP 03 (2019) 037 [arXiv: 1812.04421] [INSPIRE].

[37] I. Esteban, M.C. Gonzalez-Garcia, M. Maltoni, I. Martinez-Soler and T. Schwetz, Updated fit to three neutrino mixing: exploring the accelerator-reactor complementarity, JHEP 01 (2017) 087 [arXiv: 1611.01514] [INSPIRE]. 
[38] P.F. de Salas, D.V. Forero, C.A. Ternes, M. Tortola and J.W.F. Valle, Status of neutrino oscillations 2018: $3 \sigma$ hint for normal mass ordering and improved CP sensitivity, Phys. Lett. B $\mathbf{7 8 2}$ (2018) 633 [arXiv:1708.01186] [INSPIRE].

[39] Y. Nir, CP violation: A New era, in Heavy flavor physics: Theory and experimental results in heavy quark physics and CP-violation. Proceedings, 55th Scottish Universities Summer School in Physics, SUSSP 2001, St. Andrews, U.K., 7-23 August 2001, pp. 147-200 (2001) [hep-ph/0109090] [INSPIRE].

[40] Planck collaboration, Planck 2018 results. VI. Cosmological parameters, arXiv: 1807.06209 [INSPIRE].

[41] K. Huitu, J. Laamanen, L. Leinonen, S.K. Rai and T. Ruppell, Comparison of neutralino and sneutrino dark matter in a model with spontaneous CP-violation, JHEP 11 (2012) 129 [arXiv: 1209.6302] [INSPIRE].

[42] S.F. King, M. Muhlleitner, R. Nevzorov and K. Walz, Exploring the CP-violating NMSSM: EDM Constraints and Phenomenology, Nucl. Phys. B 901 (2015) 526 [arXiv:1508.03255] [INSPIRE].

[43] W. Buchmüller, P. Di Bari and M. Plümacher, Leptogenesis for pedestrians, Annals Phys. 315 (2005) 305 [hep-ph/0401240] [INSPIRE].

[44] J. Garayoa, S. Pastor, T. Pinto, N. Rius and O. Vives, On the full Boltzmann equations for Leptogenesis, JCAP 09 (2009) 035 [arXiv:0905.4834] [INSPIRE].

[45] F. Hahn-Woernle, M. Plümacher and Y.Y.Y. Wong, Full Boltzmann equations for leptogenesis including scattering, JCAP 08 (2009) 028 [arXiv:0907.0205] [INSPIRE].

[46] S. Davidson and A. Ibarra, A Lower bound on the right-handed neutrino mass from leptogenesis, Phys. Lett. B 535 (2002) 25 [hep-ph/0202239] [INSPIRE].

[47] M. Kawasaki, F. Takahashi and T.T. Yanagida, The Gravitino-overproduction problem in inflationary universe, Phys. Rev. D 74 (2006) 043519 [hep-ph/0605297] [INSPIRE].

[48] M.Y. Khlopov and A.D. Linde, Is It Easy to Save the Gravitino?, Phys. Lett. 138B (1984) 265 [INSPIRE].

[49] I.V. Falomkin, G.B. Pontecorvo, M.G. Sapozhnikov, M.Y. Khlopov, F. Balestra and G. Piragino, Low-energy $\bar{p}^{4}$ He annihilation and problems of the modern cosmology, gut and susy models, Nuovo Cim. (1965-1970) A 79 (1984) 193.

[50] P.S.B. Dev and A. Mazumdar, Probing the Scale of New Physics by Advanced LIGO/VIRGO, Phys. Rev. D 93 (2016) 104001 [arXiv:1602.04203] [INSPIRE]. 